Anales de Geografía de la Universidad Complutense ISSN: 0211-9803

http://dx.doi.org/10.5209/rev_AGUC.2016.v36.n1.52717

\title{
Aspectos territoriales y demográficos de la Red de Espacios Naturales Protegidos de Castilla-La Mancha: 1978-2014
}

\author{
Óscar Serrano Gil ${ }^{1}$ \\ Recibido: 30 de julio del 2015 / Enviado a evaluar: 23 de enero del 2016 / Aceptado: 3 de mayo del 2016
}

Resumen. La Comunidad Autónoma de Castilla-La Mancha desarrolla a partir de la aprobación de la Constitución Española todo un aparato ejecutivo y legislativo para poner en marcha sus políticas en materia de protección medioambiental. La nueva normativa (Ley 9/1999, de 26 de mayo) ha buscado la conservación y protección integral de los elementos naturales del territorio atendiendo a nuevos criterios de selección como la calidad ambiental de los ecosistemas o la excepcionalidad paisajística. La proliferación y la declaración de los nuevos espacios naturales ha dado como resultado un doble modelo geográfico y territorial caracterizado por espacios naturales ubicados en ámbitos territoriales rurales, generalmente de montaña, donde confluyen problemas complejos de despoblación y envejecimiento y espacios naturales situados en zonas con mayor dinamismo y volumen demográfico.

Palabras clave: Espacios Naturales Protegidos; Castilla-La Mancha; protección integral; demografía.

\section{[en] Territorial and demographic aspects of the Networks of Protected Natural Areas of Castilla-La Mancha: 1978-2014}

\begin{abstract}
The Autonomous Region of Castilla-La Mancha develops from the approval of the Spanish Constitution a whole executive and legislative branch to implement its policies on environmental protection. The new legislation (Law 9/1999, of 26 May) has pursued the conservation and the integral protection of the natural elements of the territory demanding to new criteria as such the environmental quality of ecosystems or the exceptional landscape. The spread and the declaration of new natural spaces have caused a double geographical and territorial model. First, natural spaces located in rural mountainous areas with depopulation and aging problems. And second, natural spaces situated in areas densely populated
\end{abstract}

Key words: Natural Protected Areas; Castilla-La Mancha; integral protection; demography.

1 Departamento de Geografía y Ordenación del Territorio. Universidad de Castilla-La Mancha.

E-mail: oscar.serranogil@gmail.com 
[fr] Aspects territoriaux et démographiques du Réseau des aires naturelles protégées de Castilla-La Mancha: 1978 -2014

Résumé. La Communauté Autonome de Castille -La Manche développe à partir de l'adoption de la Constitution espagnole tout un appareil exécutif et législatif pour mettre en oeuvre leurs politiques en matière de protection de l'environnement. La nouvelle législation (loi 9/1999, du 26 mai) a recherché la conservation et la protection intégrale des éléments naturels du territoire en fonction de nouveaux critères de sélection tels que la qualité environnementale des écosystèmes ou le caractère exceptionnel du paysage. La prolifération et la déclaration des nouveaux espaces naturels a entraîné un double modèle géographique et territorial caractérisé par des espaces naturels situés dans des domaines territoriales rurales où se retrouvent les problèmes complexes de dépeuplement et vieillissement des zones où sont localisés et des espaces naturels situés dans des zones à plus grand volume et de dynamisme démographique et de la population.

Mots clés: Espaces naturels protégés; Castille-La Manche; protection intégrale; démographie.

Cómo citar. Serrano Gil, O. (2016): Aspectos territoriales y demográficos de la Red de Espacios Naturales Protegidos de Castilla-La Mancha: 1978-2014. Anales de Geografía de la Universidad Complutense, 36(1), 149-171.

Sumario. 1. Introducción. 2. Objetivos y metodología. 3. Reclasificaciones y primeras declaraciones (1978-1989). 4. Segunda etapa: Consolidación del modelo autonómico (1990-2000). 5. Tercera etapa: Desarrollo y momento (2001-2014). 6. Conclusiones. 7. Bibliografía.

\section{Introducción}

Hasta la fecha de la aprobación de la Constitución Española, en Castilla-La Mancha, las primeras declaraciones de Espacios Naturales Protegidos (ENP) de principios del siglo XX responden a los modelos y criterios del momento histórico: el gusto por los ecosistemas frágiles (lagunas de Ruidera) y las formas caprichosas y sorprendentes del roquedo (Ciudad Encantada). El siguiente período histórico se caracterizará por el dominio de la actividad cinegética y este planteamiento estará en vigor hasta la Ley 15/1975, de 2 de mayo, de Espacios Naturales Protegidos (Jefatura del Estado, 1975) siendo transformado a raíz del traspaso de competencias en materia de protección de la naturaleza a las Comunidades Autónomas.

Castilla-La Mancha, como muchas otras regiones, comienza a asumir las transferencias en materia de protección medioambiental (protección de la naturaleza, conservación, declaración de ENP, etc.) recogidas en dicha norma. Este nuevo régimen constitucional, añadido al creciente empuje de los movimientos ecologistas supondrá, un notable espaldarazo para la política de conservación, con la aplicación de modelos y nuevos conceptos en la protección (Casado de Otaloa, 1990). El modelo territorial emergido de la Constitución supone una descentralización incipiente a favor de los nuevos entes territoriales (Comunidades Autónomas) adquiriendo más protagonismo político y de gestión (Mulero Mendigorri, 2002) en esta materia. A partir de este punto, se desarrolla el aparato legislativo autonómico poniendo en marcha la normativa propia en ámbitos diversos (protección medioambiental, ENP, gestión forestal, etc.) (García Rayego, 1995). Todo ello repercutirá positivamente 
materializándose en un incremento considerable de la superficie declarada y protegida a partir de este momento en todas las Comunidades Autónomas (Gómez Mendoza, 1999) atendiendo a nuevos criterios de selección para elegir los futuros ENP abarcando, en la medida de lo posible, una protección global aunando la diversidad de elementos de relieve, fruto de la evolución geológica de Castilla-La Mancha, pero también otros principios a la hora de seleccionar los nuevos ENP. La diversidad de espacios, de materiales y de paisajes que configuran el territorio regional conforman un auténtico repertorio paisajístico de interior (Mata Olmo, 2011) que ha cristalizado en la cantidad de elementos morfoestructurales que conforman estos paisajes incluyendo desde sistemas montañosos de plegamiento de cobertera a campos volcánicos inactivos.

No hay que olvidar que buena parte de estos ENP se han localizado en áreas de montaña, especialmente los más extensos, caracterizados por un descenso progresivo de la población de sus municipios y un marcado carácter rural.

\section{Objetivos y metodología}

El establecimiento de la nueva normativa en materia de protección del medio ambiente en Castilla-La Mancha supone un hito fundamental: el aumento de la superficie protegida en esta Comunidad Autónoma pasando de casi 6.000 hectáreas en el año 1978 a más de 600.000 en el año 2014. Esto supone un incremento sustancial no sólo en la superficie sino también en el número y la diversidad de nuevas figuras emanadas de la Ley 9/1999 (Ruiz Martínez, 2001) atendiendo a criterios geomorfológicos, hidrológicos y biogeográficos para la conservación y preservación de los ecosistemas.

Así mismo, la evolución de la Red de ENP parece presentar alguna correlación interesante entre su localización en el territorio regional y algunos aspectos demográficos dominando un doble modelo con características bien diferentes: por un lado, los ecosistemas protegidos en territorios rurales tradicionales con densidades medias de población y en donde, generalmente se encuentran núcleos de población bien comunicados y con dinamismo social y económico; y por otro, los espacios protegidos que se sitúan en comarcas rurales con graves dificultades de vertebración, con bajísimos niveles de densidades de población que corresponden con zonas montaña que superan los $1.000 \mathrm{~m}$. de altitud. En líneas generales, se puede afirmar que las comarcas montañosas castellano-manchegas (montes de Toledo, Serranía de Cuenca, Sierra Norte de Guadalajara, Sierra Morena, Valle de Alcudia, Sierra de Alcaraz, Sierra de San Vicente) se perfilan como los territorios que cuentan con las mayores extensiones de superficie protegida pero con menor presencia humana en el conjunto de la Comunidad Autónoma. El alto grado de conservación de algunos de estos ecosistemas añadido a la escasa presencia humana los ha convertido en "espacios de protección estrella” para sus escasos habitantes percibiendo en cada ENP un marchamo de calidad diferencial y proyectando sobre él las esperanzas de unas comarcas que acusan cada vez más la pérdida de efectos y la falta de recambio generacional. 
En cambio, la comarca central de La Mancha, los municipios próximos a las capitales de provincias o limítrofes con la Comunidad Autónoma de Madrid (la Sagra, el corredor del Henares), sin olvidar otras comarcas como la Manchuela, presentan valores, en conjunto, mucho más positivos demográfica y socialmente con densidades de población mayores pero con superficies protegidas menores asociadas a una amplia gama de figuras (reservas naturales, microrreservas, monumentos naturales, etc.).

Desde el punto de vista metodológico, en la investigación se han utilizado fuentes de carácter indirecto como las revisiones bibliográficas sobre el tema así como los accesos vía web necesarios a páginas de información.

Para la elaboración de los datos estadísticos se ha recurrido a la utilización del Padrón continuo de habitantes para Castilla-La Mancha para varios años procedente del Instituto Nacional de Estadística (INE), varios censos de Población de diversos años (INE), datos demográficos, económicos y sociales procedentes del Instituto de Estadística de Castilla-La Mancha, el atlas socioeconómico de Castilla-La Mancha (año 2011) publicado por esta misma institución y los datos económicos y sociales a escala municipal que facilita la entidad financiera Caja España en formato .pdf.

Entre las fuentes cartográficas cabe destacar la base cartográfica digital (municipios, provincias y comunidades autónomas españolas) procedente del INE (año 2014), la cartografía y los datos de la Red de ENP de Castilla-La Mancha suministrada por la Consejería competente y cartografía procedentes del Ministerio de Agricultura, Alimentación y Medio Ambiente a través del Banco de Datos de la Naturaleza (parques nacionales, Red Natura, etc.).

\section{Reclasificaciones y primeras declaraciones (1978-1989)}

Castilla-La Mancha nace como Comunidad Autónoma a partir de la aprobación su Estatuto de Autonomía el 16 de agosto de 1982. Será el aldabonazo de salida para la puesta en marcha de los órganos de gobierno (ejecutivo y legislativo) que configurarán las posteriores Consejerías, bajo diversas nomenclaturas, encargadas de la gestión de la protección medioambiental.

Pero habrá que esperar hasta 1984, cuando se hagan efectivas las transferencias mediante el Real Decreto 1676/1984 de 8 de febrero (Jefatura del Estado, 1984). A partir de este año, las diversas figuras existentes en el territorio (el parque nacional de Daimiel) (Sánchez Soler y Del Moral Fernández Del Rincón, 2000) y los parques naturales (Lagunas de Ruidera y Hayedo de Tejera Negra), quedarán en manos de la Junta de Comunidades, siendo administrados por el Instituto Nacional para la Conservación de la Naturaleza (ICONA) hasta la gestación de una Consejería competente en esta materia.

En este mismo período y como consecuencia de varias reclasificaciones llevadas a cabo, el parque natural del Hayedo ampliaría su superficie hasta las 1.641 ha. (año 1979). Así mismo, también se realizaron declaraciones interesantes como en el caso de Cabañeros que se convierte en parque natural (año 1988) con una extensión inicial que superaba las 25.000 ha. 
Entre las "figuras" de nuevo cuño de estos primeros momentos destaca la puesta en marcha de los Refugios de Caza y Pesca los cuales, se conciben como lugares de salvaguarda de la fauna y flora que habita en esos ecosistemas, prohibiendo las actividades cinegéticas en sus límites. El primer Decreto de estas características, aprobado por la Consejería de Agricultura (Decreto 65/1988, de 17 de mayo) creaba los primeros Refugios de $\mathrm{Caza}^{2}$ abarcando con esta protección principalmente superficies lagunares de diversa índole.

La aprobación regional de su Ley 2/1993, de 15 de julio, de caza provocó un cambio en la condición y en la denominación de estas figuras pasando de ser denominadas Refugios de Fauna pero conservando las mismas superficies y límites que los establecidos en sus Decretos de su creación. En la mayoría de los casos, y hasta el momento actual, la evolución de la superficie ha sido progresiva ascendiendo hasta superar las 15.000 ha. Algunos de estos espacios, en aras de la Ley 9/1999, 26 de mayo, pasaron a ser reservas naturales cuya normativa propia prohíbe la caza salvo supuestos excepcionales que requieran control de alguna especie como es el caso de la laguna de Pétrola y las lagunas Grande y Chica de Villafranca de los Caballeros, entre otras.

De manera paralela, también la protección medioambiental llegaría a los cauces de los río mediante la gestación de los llamados Refugios de Pesca surgidos a raíz de la Ley 1/1992, de 7 de mayo, de pesca fluvial, la cual establecía este tipo de "figura" para conservar los cauces de los ríos. Hasta el año 1994, se habían declarado cuatro Refugios de Pesca, dos de ellos situados en la provincia de Albacete (los Chorros del río Mundo y el río Endrinales), y uno en la de Cuenca (Arroyo Almagrero) y otro en la de Guadalajara (río Pelagallinas).

En esencia, en este período autonómico los comienzos de la protección son concebidos más como un claro espíritu continuista de reclasificaciones de ENP que como un nuevo impulso en nuevas declaraciones quizás debido, en parte, al efecto prematuro de la asunción de las competencias ya que -a nivel legislativo y ejecutivodicha recepción suponía un ingente esfuerzo de todo el engranaje administrativo referente el medio ambiente y a la protección de espacios naturales. Así pues, el final de la década de los años 80 , se concluye que fue un decenio principalmente de reajuste y reclasificación en materia medioambiental por un doble motivo. Por un lado, las declaraciones previas fueron reordenadas y gestionadas por el nuevo ejecutivo regional en aras de la asunción de las competencias ambientales, y por otro lado, se desarrollaron los primeros hitos legislativos básicos apoyados en tímidas protecciones, declaraciones y reclasificaciones situándose en distintos territorios de la Comunidad Autónoma percibiéndose ya un ligero contraste especialmente demográfico entre comarcas afectadas por estas figuras.

Según la norma de aprobación, se declararon Refugios de Caza: la laguna de los Patos (Hellín), la del Alcahozo y la de la Vega (en Pedro Muñoz), la del Prado (en Pozuelo de Calatrava), la laguna de El Tobar (en Beteta) y la de Uña. 
La población ha sido uno de los más determinantes elementos a la hora de evaluar el éxito de la declaración de una nueva porción de territorio ya que un nuevo espacio natural afecta directa o indirectamente a los intereses -materiales o no- de todo un colectivo y de toda una comunidad, siendo en cierta medida los principales actores y protagonistas de todo el proceso de declaración y también de mantenimiento del ecosistema. Así pues, en este primer período histórico, el contingente poblacional que se vería afectada durante por estas figuras protegidas se situaba en torno a un $4 \%$ sobre el total.

El gráfico (figura 1) resume visualmente el porcentaje de población afectada por las Áreas de Influencia Socioeconómica (AIS ${ }^{3}$ ) por la presencia de alguno de los espacios protegidos del momento. En el año 1970, la población total regional se cifraba en 1.759.203 habitantes, de los que sólo un 4,25\% se encontraba afectado por alguna figura de protección. En cambio, y a pesar del descenso de población en la década siguiente, del total de población en Castilla-La Mancha (1.626.845 habitantes), el contingente poblacional vinculado a ENP había aumentado ligeramente (4,42\%
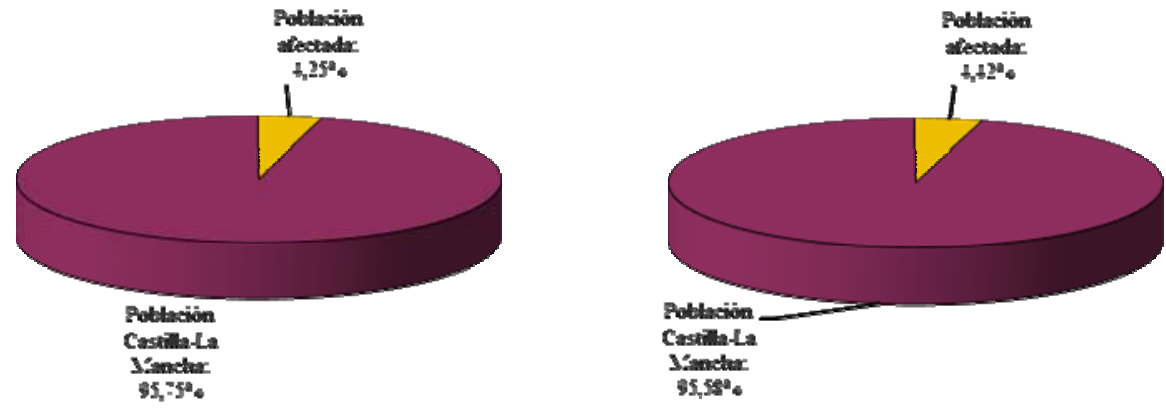

sobre el total).

Figura 1. Porcentaje de población residente afectada por el AIS de ENP (1970-1981).

Fuente: Instituto de Estadística de la Junta de Comunidades de Castilla-La Mancha. Elaboración propia.

Atendiendo al total de población relacionada con las figuras reclasificadas y declaradas encontramos algunos contrastes interesantes. Es de destacar que en el término municipal de Cantalojas en el año 1970, el volumen de población total apenas superaba los trescientos habitantes. Once años después y siguiendo la dinámica dominante de salida de población de los núcleos rurales se acusa una pérdida notable (257 habitantes) lo que supone un 19\% sobre el total. Esto explica en parte por qué el

Por Área de Influencia Socioeconómica recogemos la definición de la normativa aludiendo al conjunto de los términos municipales en cuyo territorio se encuentra situada alguna parte de un Parque nacional (Jefatura de Estado, 1982). 
decreto de reclasificación del parque natural contemplaba medidas de ayuda socioeconómica para la zona con el fin de arraigar población en una comarca rural y envejecida. Esta misma tendencia de descenso poblacional es extrapolable al caso de Cabañeros perdiendo casi mil habitantes en una década.

En cambio, en los dos restantes espacios naturales, el contingente de población es mayor. Hay que tener en cuenta que la localización de núcleos con mayor número de habitantes en el territorio de Castilla-La Mancha se concentra, principalmente en la llanura manchega, y en comarcas con dinamismo poblacional y demográfico. En las Tablas de Daimiel, que, a pesar de la disminución en el número total de municipios de su AIS, en el año 1991, contaba con casi 30.000 habitantes y las lagunas de Ruidera que mantienen un peso demográfico superior a los 14.000 habitantes para el conjunto de pueblos de su AIS, son los ejemplos más sobresalientes. No obstante, el hecho de contar con población en las AIS es esencial para el equilibrio de los espacios naturales: recursos naturales e impacto humano. Por ello, no se puede valorar de igual forma un espacio con baja densidad de población, en donde muy probablemente los valores ecológicos se mantienen más inalterados, que los espacios con mayor volumen poblacional donde soportan mayor impacto humano social y económico.

Tabla 1. Población afectada por los ENP (1978-1991)

\begin{tabular}{|c|c|c|c|c|c|}
\hline \multirow[t]{2}{*}{ ENP } & \multirow[t]{2}{*}{$\begin{array}{l}\text { Superficie } \\
\text { (ha.) }\end{array}$} & \multirow[t]{2}{*}{$\begin{array}{c}\text { Municipios que conforman el } \\
\text { AIS }\end{array}$} & \multicolumn{3}{|c|}{$\begin{array}{l}\text { Evolución de la población } \\
\text { (habitantes) }\end{array}$} \\
\hline & & & 1970 & 1981 & 1991 \\
\hline $\begin{array}{l}\text { Hayedo de } \\
\text { Tejera Negra }\end{array}$ & 1.391 & Cantalojas & 317 & 257 & 154 \\
\hline $\begin{array}{l}\text { Lagunas de } \\
\text { Ruidera }\end{array}$ & 3.772 & $\begin{array}{l}\text { Argamasilla de Alba, Alhambra, } \\
\text { Villahermosa, Ossa de Montiel y } \\
\text { Ruidera }\end{array}$ & 16.320 & 13.334 & 14.402 \\
\hline $\begin{array}{l}\text { Tablas de } \\
\text { Daimiel }\end{array}$ & 1.928 & Ciudad Real $^{4}$ & 58.053 & 58.350 & $29.171^{5}$ \\
\hline Cabañeros & 25.615 & $\begin{array}{l}\text { Alcoba, Retuerta del Bullaque, Navas } \\
\text { de Estena y Horcajo de los Montes }\end{array}$ & 4.787 & 3.775 & $6.471^{6}$ \\
\hline Total & 32.706 & & 74.690 & 71.941 & 29.171 \\
\hline
\end{tabular}

Fuente: INE. Elaboración propia.

4 Según se establece en la Ley 25/1980, de 3 de mayo, de reclasificación del Parque nacional, el AIS, en esa fecha estaría compuesta por los municipios de Daimiel, Villarrubia de los Ojos, Torralba de Calatrava, Manzanares, Carrión de Calatrava, Bolaños de Calatrava y Llanos del Caudillo. No obstante, esta AIS se ha reducido notablemente afectando sólo a dos municipios de la provincia de Ciudad Real (Daimiel y Villarrubia de los Ojos) aunque en la Zona Periférica de Protección habría que incluir las 30 hectáreas pertenecientes al término municipal de Torralba de Calatrava.

5 Total calculado atendiendo a los tres municipios del AIS (Daimiel, Villarrubia de los Ojos y Torralba de Calatrava).

6 A partir de la aprobación de la Ley 33/1995, 20 de noviembre, se amplía el conjunto de municipios del AIS con Hontanar y Los Navalucillos. 


\section{Segunda etapa: Consolidación del modelo autonómico (1990-2000)}

Los comienzos de este decenio mantienen las reclasificaciones de espacios naturales aunque al final de esta década, y debido al nuevo marco de protección para todo el territorio regional (Ley 9/1999, de 26 de mayo) (Presidencia de Junta de Comunidades de Castilla-La Mancha, 1999), el incremento en declaraciones y por ende en superficie protegida será notorio. En esa línea de reclasificaciones, cabe destacar el Decreto 34/1990, de 13 de marzo, que reajusta las superficies de los tres parques naturales situados en Castilla-La Mancha hasta ese momento: las lagunas de Ruidera (3.772 ha.), el hayedo de Tejera Negra (1.641 ha.) y Cabañeros (25.615 ha.).

Como consecuencia de la aprobación de la normativa regional específica, comienzan a proliferar las declaraciones de espacios, teniendo en cuenta las figuras propuestas por la Ley. Por la Ley 33/1995, de 20 de noviembre, se declara el espacio de Cabañeros como parque nacional ampliando su extensión (40.855,98 ha.) dados sus singulares valores naturales y el interés general que su conservación implicaba. En ese mismo año se elabora el Plan de Ordenación de los Recursos Naturales $\left(\mathrm{PORN}^{7}\right)$ para la zona de los Montes de Toledo (Cabañeros-Rocigalgo) aprobado por Decreto (Presidencia de Junta de Comunidades de Castilla-La Mancha, 1995) y que posteriormente declarado nulo en 2003 por el Tribunal Supremo.

En el transcurso de la década de los noventa, se materializaron algunos hitos normativos importantes. Por un lado, la aprobación del Plan de Conservación del Medio Natural (1994-1995) que servirá de instrumento base y previo a la ley específica sobre espacios protegidos en Castilla-La Mancha a partir del que se actuará como marco de referencia (García Rayego, 2001). Será la herramienta de trabajo a corto, medio y largo plazo para la conservación de los recursos naturales, su gestión y conservación de la naturaleza convirtiéndose en la base de partida de la posterior legislación de ENP de Castilla-La Mancha. Por otro lado, la aprobación de varias órdenes de la Consejería de Agricultura y Medio Ambiente, proponiendo ayudas para la adopción de prácticas agrícolas compatibles con la conservación de las aves esteparias de diecinueve espacios del territorio regional. Y finalmente, la Ley 9/1999, de 26 de mayo, de Conservación de la Naturaleza en Castilla-La Mancha de 1999 que obedece a la normativa estatal (Ley 4/1989, de Conservación de los Espacios naturales y de la Flora y de la Fauna) (Jefatura del Estado, 1989).

La Ley autonómica se considera el punto de inflexión para la consolidación de una Red regional de espacios protegidos en Castilla-La Mancha (García Rayego y Serrano de la Cruz Santos-Olmo, 2007). Entre los objetivos que persigue cabe destacar: el establecimiento de las categorías de espacios protegidos en Castilla-La Mancha en virtud de la variedad de los territorios, espacios y situaciones reconocidas y existentes (siete categorías); el desarrollo pleno de las competencias en materia de ENP

El Plan de Ordenación de los Recursos Naturales es la herramienta básica para garantizar la conservación, la restauración y la puesta en valor de todos los recursos naturales (Ley 9/1999, de 26 de mayo). 
(declaración, ampliación, anulación); el procedimiento, contenido y alcance de los Planes Rectores de Uso y Gestión (PRUG ${ }^{8}$ ), entre otros. La nueva Ley fijará normas para la protección, conservación, restauración, gestión y mejora de los recursos naturales y procesos ecológicos esenciales en la Región (Donaire Jiménez, 2005).

Tabla 2. Evolución de la población total afectada por Parques Naturales y Nacionales (19912011)

\begin{tabular}{|c|c|c|c|c|c|}
\hline \multirow{2}{*}{ Año } & \multirow{2}{*}{ ENP } & \multirow{2}{*}{$\begin{array}{c}\text { Superficie } \\
\text { (ha.) }\end{array}$} & \multicolumn{3}{|c|}{ Población } \\
\hline & & & Año 1991 & Año 2001 & Año 2011 \\
\hline 1980 & $\begin{array}{l}\text { Parque nacional Tablas } \\
\text { de Daimiel }\end{array}$ & 1.928 & 29.171 & 29.809 & 29.769 \\
\hline 1990 & $\begin{array}{l}\text { Parque natural las } \\
\text { Lagunas de Ruidera }\end{array}$ & 3.772 & 14.402 & 13.265 & 13.937 \\
\hline 1990 & $\begin{array}{l}\text { Parque natural Hayedo } \\
\text { de Tejera Negra }\end{array}$ & 1.641 & 154 & 156 & 172 \\
\hline 1995 & $\begin{array}{l}\text { Parque nacional } \\
\text { Cabañeros }\end{array}$ & $40.855,98$ & 6.471 & 6.137 & 6.011 \\
\hline 2000 & $\begin{array}{l}\text { Parque natural Alto } \\
\text { Tajo }\end{array}$ & 105.721 & 6.404 & 6.519 & 5.435 \\
\hline & Total & $153.917,98$ & 53.632 & 55.886 & 55.324 \\
\hline
\end{tabular}

Fuente: INE. Elaboración propia.

Además de la nueva clasificación recogida en las categorías (parque nacional, parque natural, monumento natural, reserva natural, microrreserva, reserva fluvial paisaje protegido y paraje protegido) también aportó un nuevo aspecto: la creación de una Red regional de espacios (Red de Áreas Protegidas) conformada por las categorías de los ENP y las Zonas Sensibles ${ }^{9}$ (Serrano de la Cruz Santos-Olmo, 2014).

Durante esta etapa la proliferación de ENP en el territorio regional se materializó en las declaraciones de un parque natural (Alto Tajo), tres reservas naturales (Hoces

8 Recoge una serie de directrices sectoriales dirigidas a los diversos organismos actuantes en el espacio (administraciones, instituciones, asociaciones,...) (Gómez Orea y Rubio Blanco, 2000: 165).

9 Respecto a las Zonas Sensibles, responden a espacios naturales que pueden albergar realidades muy variopintas. En este sentido, se considerarán tales Zonas Sensibles: las ZEC (Zonas de Especial Conservación procedentes de la Directiva Hábitats 92/43/CEE), las figuras procedentes de la Red Natura 2000 - ZEPAs (Zonas de Especial Protección para las aves silvestres) y LICs (Lugares de Interés Comunitario), las Áreas Críticas, surgidas de la aplicación de los planes de conservación de especies amenazadas (Martín Herrero, 2003), las Áreas forestales, los Refugios de Pesca, los Refugios de Fauna y aquellas otras que declare el Consejo de Gobierno por su relevante función como corredores biológicos, o por resultar preciso para el cumplimiento de normas o convenios de carácter regional, nacional o internacional. 
del Cabriel, Complejo Lagunar de Alcázar de San Juan y la Laguna del Salicor), diez monumentos naturales y dos microrreservas.

Respecto a la población local residente y afectada por estas nuevas figuras de protección -Parques Nacionales y naturales- el comportamiento de sus AIS presenta aspectos interesantes.

En el conjunto de AIS se observan tres pautas diferentes. En primer lugar, el mantenimiento de la población con un ligero repunte demográfico (caso de Cantalojas, en el Hayedo de Tejera Negra) probablemente porque este municipio "habría tocado fondo" después de perder más del 50\% de la población desde el año 1960. En segundo lugar, la tendencia del descenso poblacional, aspecto más acusado en unos casos que en otros, propio de la comarcas de Cabañeros y del Alto Tajo. Y finalmente, la tercera pauta correspondería con un ligero crecimiento del total de habitantes en la comarca dejándose sutilmente en los casos de las Lagunas de Ruidera y de las Tablas de Daimiel.

Si analizamos los datos a escala regional, la población vinculada en las AIS en esta etapa (1991-2001) aumentó en más de dos mil personas pasando de más de 53.000 a casi 56.000 a la par que hubo un incremento poblacional total en Castilla-La Mancha pasando de 1.651.833 habitantes (año 1991) a 1.755.053 habitantes (año 2001). Este hecho refleja que, en cierto modo, conforme aumentan las declaraciones en el territorio también asciende el número de personas afectadas por el AIS de un ENP. No obstante, la declaración de estos espacios sigue quedando al margen de la dinámica poblacional dominante cuando no protegen territorios rurales y con serios riesgos de viabilidad socioeconómica y demográfica a medio plazo. En ese sentido habría que destacar cómo los ENP declarados y situados en las zonas montañosas de Castilla-La Mancha (Sistema Ibérico, Montes de Toledo, etc.) se posicionan como los territorios más extensos en superficie pero también los de menor presencia demográfica como lo recogen las extensas superficies de Cabañeros (más de 40.000 ha.) y del Alto Tajo (más de 100.000 ha.) frente a los situados en la comarca central de la Región (Tablas de Daimiel) y en zonas de dinamismo demográfico importante (lagunas de Ruidera).

\section{Tercera etapa: Desarrollo y momento (2001-2014)}

En Castilla-La Mancha se asiste a la consolidación del modelo regional caracterizado principalmente por una eclosión de declaraciones de ENP. Hasta el momento actual, el ejecutivo regional había ido configurando y articulando la normativa en materia medioambiental acabando con la aprobación de la Ley propia. Será ahora, y atendiendo especialmente, a las características propias del territorio, de diversidad de paisajes, de ecosistemas, de variedad de fauna y flora, cuando decida aumentar las declaraciones y por ende, la superficie protegida por alguna de sus figuras. Aunque el período abarca hasta el año 2014, las últimas declaraciones se realizan en el año 2011.

El incremento de nuevos espacios y en consecuencia, de extensiones protegidas, será prominente (figura 2). Aunque ha habido declaraciones todos los años siguiendo 
una tendencia alcista no todos los años serán igual de "productivos". Respecto al número total de ENP en esta última etapa se declararán 91 espacios, los cuales, sumados a los que previamente habían sido declarados desde el año 1933 en adelante, elevan a 110 el total de ENP a fecha del año 2014. Especialmente, los cinco primeros años (2001-2006) fueron prolíficos en las declaraciones (casi 80) mientras que la corriente a partir de ese momento ha sido claramente a la baja en comparación con el quinquenio anterior.

Figura 2. Superficie protegida por año y número de declaraciones (2001-2014)

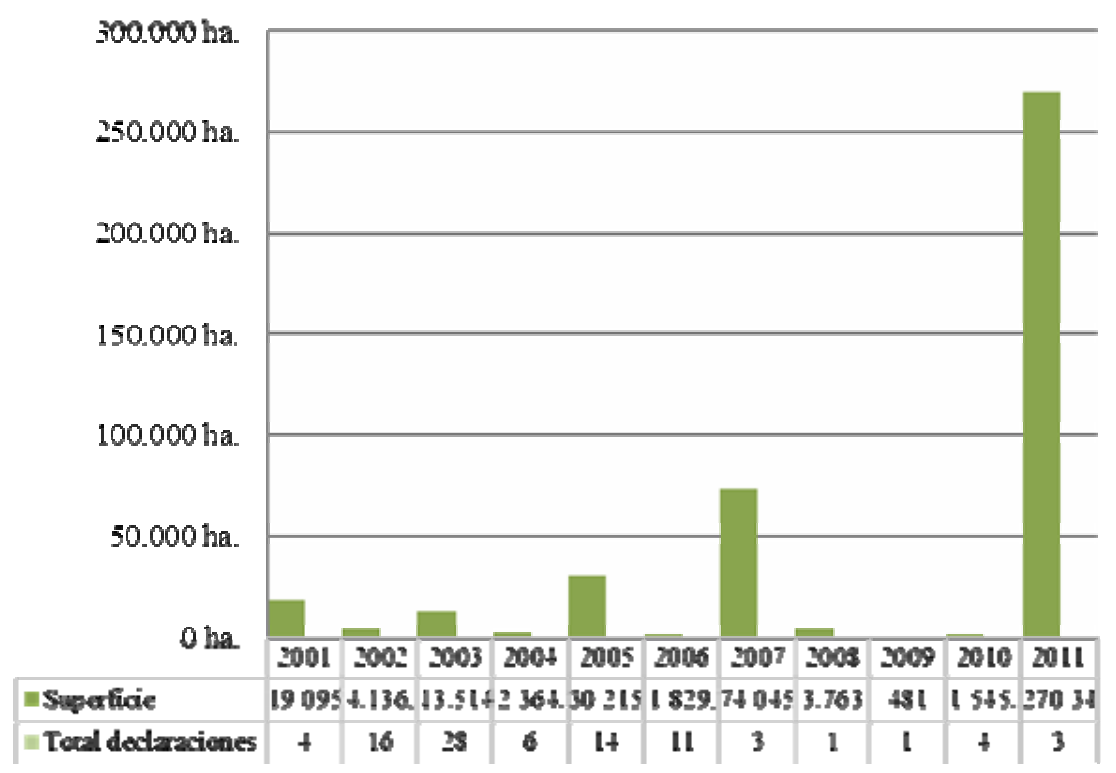

Fuente: Junta de Comunidades de Castilla-La Mancha. Elaboración propia.

En la tercera etapa, el incremento ha sido sin duda el más sobresaliente aunque con altibajos desde 2001. Entre los años 2001 y 2007 (Serrano Gil y Vázquez Varela, 2007) la tendencia general fue el aumento de superficie declarada intercalándose años con menor impacto (2002, 2004 y 2006). En el año 2001 más del 95\% de la extensión protegida corresponde con el monumento natural de Palancares y Tierra muerta (18.078 ha.). Al año siguiente (2002), mayoritariamente se declaran microrreservas (11) de las 16 figuras protegidas. En el año 2003, será el año estrella desde el punto de vista del total de figuras declaradas (28) aunque en superficie apenas superen las 13.500 ha. destacando las más de 8.300 ha. del parque natural de Barranco del río Dulce en la provincia de Guadalajara. Al año siguiente (2004), habrá 6 declaraciones y más de 2.300 ha. El año 2005 supone otro hito importante (más de 30.000 ha.) de las que al menos 19.192 ha. corresponden con el parque natural de los Calares del río 
Mundo en la provincia de Albacete. Once declaraciones suman más de 1.800 ha. (año 2006) de las que siete de ellas corresponden con reservas naturales. Y finalmente, en el año 2007, la declaración del parque natural de la Serranía de Cuenca marca un hito ascendente importante en este período (73.726 ha.).

Desde el año 2008 al momento actual encontramos una evolución dispar. Durante los tres primeros años (2008, 2009 y 2010) sólo se han declarado seis figuras (cuatro de ellas en el año 2010). En el año 2008, sólo se declara el monumento natural del macizo volcánico de Calatrava (3.763 ha.); en el año 2009 otro monumento, el volcán de Piedrabuena (481 ha.); en el año 2010, las más de 1.500 ha. declaradas se reparten entre la microrreserva del complejo lagunar del río Moscas, la reserva fluvial del río Guadalmez, y los monumentos naturales de las barrancas de Castrejón y Calaña y el volcán del Alhorín. Y finalmente, en el año 2011, se declaran dos parques naturales, el de Sierra Norte de Guadalajara (117.898 ha.) y el del Valle de Alcudia y Sierra Madrona (149.463 ha.) además de la reserva natural de las Lagunas y Albardinales del Cigüela.

Asimismo, la figura que más se ha utilizado en las declaraciones de ENP ha sido la de microrreserva con 48 en total (43\%), seguida de la reserva natural con 24 declaraciones (22\%), le sigue los 24 monumentos naturales (21,43\%), los 7 parques naturales $(6,36 \%), 6$ reservas fluviales (5,36\%), un paisaje protegido (1\%) y ningún paraje protegido declarado.

Figura 3. Superficie ENP Castilla-La Mancha.

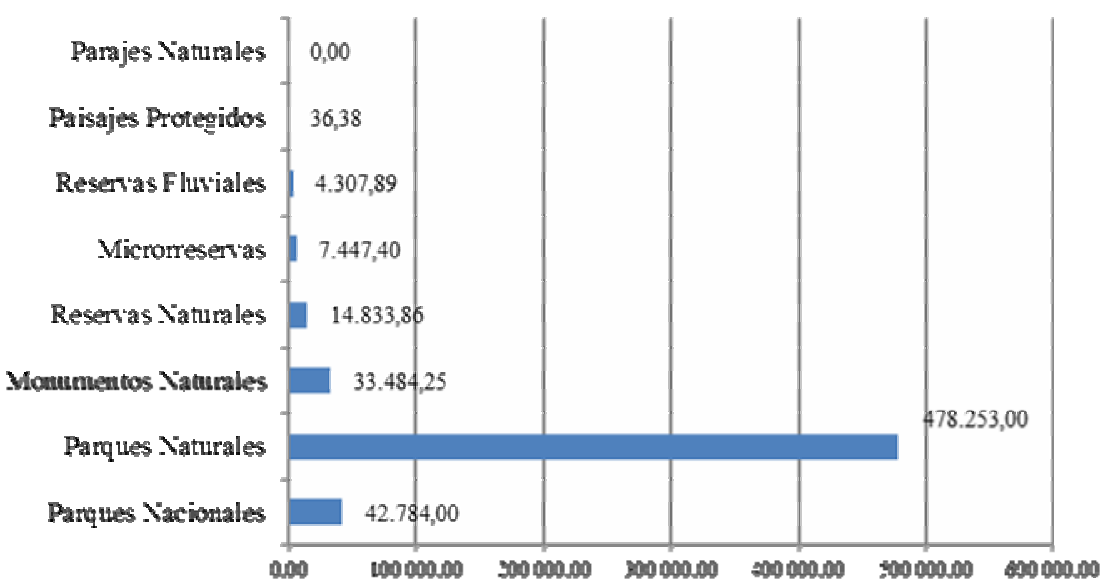

Fuente: Junta de Comunidades de Castilla-La Mancha. Elaboración propia.

Si cuantitativamente la figura de la microrreserva es la que más se utilizado desde la puesta en marcha de la normativa y legislación autonómica, paradójicamente desde el punto de vista superficial sólo afecta a algo más de 7.400 ha., mientras que en el lado opuesto situaríamos a los parques naturales ( 7 declarados) que en cambio suman 
más de 475.000 ha. (figura 3), seguido de la superficie declarada en los parques nacionales con 42.784 ha., después estarían los monumentos naturales con más de 33.000 ha.; las reservas naturales con más de 24.000 ha., las reservas fluviales que presentan algo más de 4.300 ha. y el paisaje protegido que testimonialmente presenta algo más de 35 ha.

En la evolución superficial por años (figura 4) encontramos comportamientos diversos. Por un lado, hasta el año 1980 caracterizado por ser un período de reclasificaciones y de reestructuración normativa (Ley 15/1975, de 2 de mayo), la protección se mantuvo en un nivel constante. A partir de esa fecha, la pauta comienza un ligero aumento que coincide con superficies extensas de Parques nacionales y naturales -Cabañeros (1995), Alto Tajo (2000), Calares del Mundo (2005), río Dulce, Serranía de Cuenca (2007), Sierra Norte de Guadalajara y Valle de Alcudia ${ }^{10}$ (2011). Y finalmente, encontraríamos un comportamiento estable pero progresivo que supone una ligera crecida de la superficie en los últimos años (2007-2010).

Figura 4. Incremento superficial acumulado por año (1978-2014)

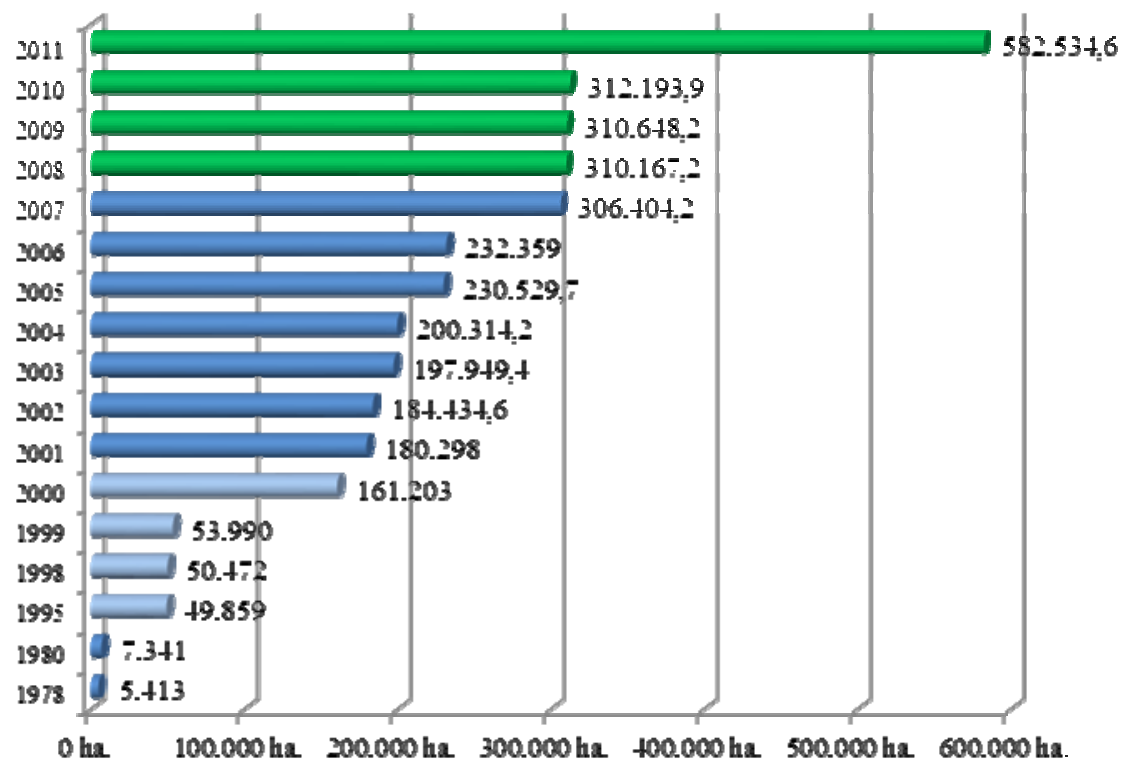

Fuente: Junta de Comunidades de Castilla-La Mancha. Elaboración propia.

10 Ambos espacios están pendientes de la resolución de sendos recursos de inconstitucionalidad por algunos artículos en sus normas de declaración. 
En este sentido, y atendiendo a la extensión de la superficie declarada en el último período (2011-2014), también la población local vinculada residente en las AIS de los principales ENP ha seguido patrones diversos (tabla 3). En el año 2009, el total de población vinculada se situaba en algo más de 86.000 habitantes aunque hay que tener en cuenta que para esa fecha no estaban declarados todavía los dos últimos Parques Naturales (Valle de Alcudia y Sierra Norte de Guadalajara) que suponen algo más de 15.000 habitantes. Sin embargo, la tendencia de ascenso desciende desde este misma fecha (2009) y en el año 2011, el total de población se sitúa en los 85.000 habitantes continuándose la línea descendente hasta el año 2013 con 83.073 habitantes. Si comparamos los datos de los últimos dos años (2011-2013) sólo el caso del AIS de las Tablas de Daimiel ha aumentado ligeramente su volumen poblacional, mientras que el resto de AIS muestran claramente pérdidas siendo especialmente acusado en las comarcas de montaña de la Región (Cabañeros, Alto Tajo, Serranía de Cuenca, etc.) (Serrano Gil, 2009).

Tabla 3. Población total afectada por Parques Naturales y Nacionales (2009-2013)

\begin{tabular}{|c|c|c|c|c|}
\hline ENP & $\begin{array}{l}\text { Año } \\
2009\end{array}$ & $\begin{array}{l}\text { Año } \\
2011\end{array}$ & $\begin{array}{l}\text { Año } \\
2013\end{array}$ & $\begin{array}{c}\text { Densidad pobla- } \\
\text { ción } 2013 \text { (hab./ } \\
\text { km²) }^{2}\end{array}$ \\
\hline Tablas de Daimiel $^{11}$ & 29.718 & 29.769 & 29.778 & 41 \\
\hline Cabañeros & 6.084 & 6.011 & 5.652 & 3,1 \\
\hline Lagunas de Ruidera & 14.076 & 13.937 & 13.699 & 10,6 \\
\hline Hayedo Tejera Negra $^{12}$ & 152 & 172 & 158 & 1 \\
\hline Alto Tajo & 5.405 & 5.435 & 5.037 & 1,6 \\
\hline Río Dulce & 5.925 & 5.834 & 5.564 & 5,3 \\
\hline Calares del Mundo y de la Sima & 7.013 & 6.791 & 6.426 & 8,3 \\
\hline Serranía de Cuenca $^{13}$ & 1.945 & 1.950 & 1.744 & 3,6 \\
\hline Valle de Alcudia & 11.765 & 11.516 & 11.281 & 3,8 \\
\hline Sierra Norte Guadalajara & 4.033 & 3.985 & 3.734 & 2,3 \\
\hline Total & 86.116 & 85.400 & 83.073 & \\
\hline Castilla-La Mancha & & 2.106 .331 & 2.094.391 & 26,7 \\
\hline
\end{tabular}

Fuente: INE. Elaboración propia.

11 Hemos tenido en consideración la ampliación de superficie del parque nacional de las Tablas de Daimiel en más de mil cien hectáreas, lo que supone, sumado a lo anterior: 3.030,5 ha.

12 Hemos añadido el parque natural del Hayedo de Tejera Negra para realizar un análisis más completo de la población. Ahora bien, a partir de 2011, este parque queda subsumido en otro más amplio (Sierra Norte de Guadalajara).

13 No se ha incluido el término municipal de la ciudad de Cuenca. 
El año 2011 a pesar del incremento considerable para la red en términos espaciales (más de 260.000 ha.) desde el punto de vista demográfico no ha sido igual ya que, aunque ha habido un aumento de más de 15.000 personas, estos extensos parques naturales se sitúan en territorios con muy bajas densidades de población $\left(3,8 \mathrm{hab} / \mathrm{km}^{2}\right.$ y 2,3 hab. $/ \mathrm{km}^{2}$, respectivamente) siguiendo la línea dominante de los ENP situados en los rebordes montañosos.

Si extrapolamos estos datos al valor medio de la densidad de población en Castilla-La Mancha sólo el AIS de Tablas de Daimiel (41 hab./ $\mathrm{km}^{2}$ ) se sitúa por encima de ese dato. Le sigue el AIS de las Lagunas de Ruidera $\left(10,6 \mathrm{hab} . / \mathrm{km}^{2}\right)$ con un valor demográfico nada desdeñable comparándolo con el resto de AIS de los distintos parques naturales. Así pues, y salvo la excepción del AIS de los Calares del río Mundo $/ 8,3 \mathrm{hab} . / \mathrm{km}^{2}$ ) todas las AIS de los parques naturales se sitúan por debajo de los 6 hab. $/ \mathrm{km}^{2}$ indicando que estaríamos ante auténticos "desiertos" poblacionales especialmente en algunos casos como el Alto Tajo y de la Sierra Norte de Guadalajara. Este análisis arroja que, desde el punto de vista demográfico, los ENP que se sitúan en las comarcas de mayor altitud y con presencia de montaña de Castilla-La Mancha (Alto Tajo, Serranía de Cuenca, Sierra Norte de Guadalajara, Cabañeros, etc.) presentan pesos demográficos muy bajos en comparación con los situados en otros territorios, especialmente del interior de la Comunidad Autónoma, debido en parte al dinamismo poblacional, económico y social de estos espacios. Parece evidente pensar que la marginalidad y la ruralidad de los territorios vinculados a la dorsal Ibérica no es meramente espacial sino que tiene su trasunto en su situación socioeconómica y, por tanto, en su situación de desierto población, envejecimiento, desvertebración territorial y atraso crónico que arrastran buena parte de estas comarcas.

Por tanto, esta tercera etapa supone, en cifras absolutas, un aumento considerable tanto de nuevas declaraciones como de superficie protegida por el elenco de figuras recogidas al amparo de la normativa regional. Así, y a modo de resumen, respecto al total de ENP que componen la Red de Áreas Protegidas casi 600.000 ha. han sido protegidas por alguna figura (tabla 4).

Tabla 4. Tabla resumen ENP Castilla-La Mancha.

\begin{tabular}{|c|l|r|}
\hline $\mathbf{N}^{\mathbf{0}}$ Total espacios & \multicolumn{1}{|c|}{ Figura } & \multicolumn{1}{c|}{ Superficie (ha.) } \\
\hline 7 & Parques naturales & 478.253 \\
\hline 2 & Parques nacionales & $43.886,5$ \\
\hline 24 & Monumentos naturales & $33.484,3$ \\
\hline 22 & Reservas naturales & $14.833,9$ \\
\hline 48 & Microrreservas & $7.447,4$ \\
\hline 6 & Reservas fluviales & $4.307,9$ \\
\hline 1 & Paisajes protegidos & 36,4 \\
\hline $\mathbf{1 1 0}$ & & $\mathbf{5 8 2 . 2 4 9}$ \\
\hline
\end{tabular}

Fuente: Junta de Comunidades de Castilla-La Mancha. Elaboración propia. 
Figura 5 . ENP y densidad de población (2013).

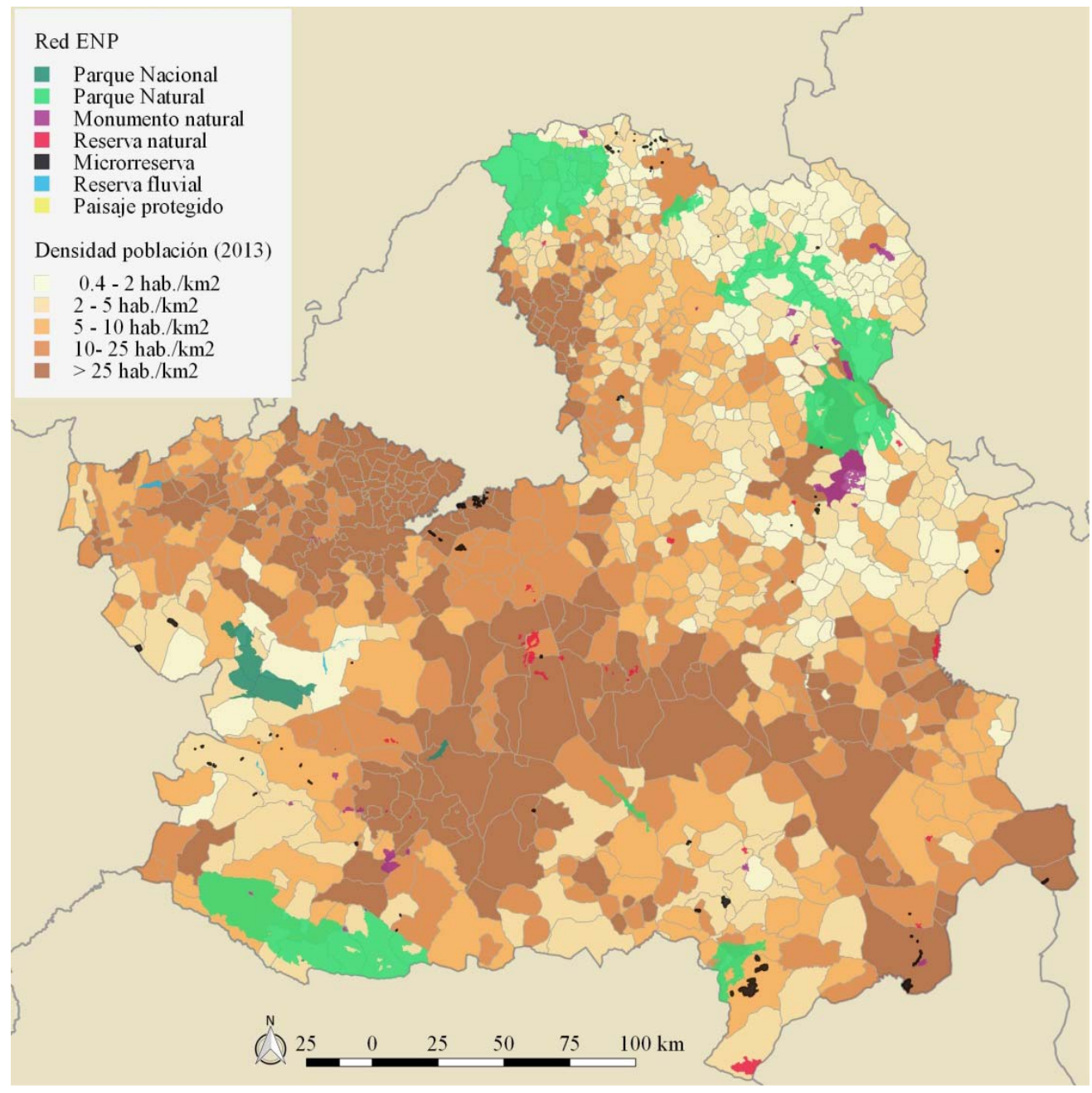

Fuente: Junta de Comunidades de Castilla-La Mancha, INE y Ministerio de Agricultura, Alimentación y Medio Ambiente. Elaboración propia.

A esta cifra habría que sumarle las distintas protecciones de diverso carácter (internacional, regional, etc.) que aglutinan algunos ENP. Así, la Red Natura 2000 contaría con más de 2.500 .000 ha. protegidas por sus 110 espacios ZEPAs o LICs; las Áreas Críticas establecidas por la Administración Regional suponen una extensión superior al millón de hectáreas protegidas y vinculadas a la importancia de las especies de flora y fauna (Martín Herrero, 1995); los ocho humedales incluidos en el convenio Ramsar cuentan con casi 10.000 ha. protegidas; la reserva de la Biosfera "Mancha Húmeda”, de alto valor ambiental (Ruiz Pulpón, Serrano De La Cruz M. A. 
et al., 2010), que inicialmente contaba con unas 25.000 ha. ha sido ampliada hasta casi 420.000 ha. acogiendo en su interior 76 lagunas y zonas de dominio público hidráulico de treinta y tres cauces fluviales (Ministerio De Agricultura; Alimentación y Medio Ambiente, 2014); y los 28 refugios representan algo más de 27.000 hectáreas protegidas para la conservación de los ecosistemas fluviales principalmente. Conviene recordar que este solapamiento de categorías provoca que buena parte de los espacios protegidos se encuentren afectados por protecciones procedentes de diversas esferas (regional, nacional, europea,...) lo cual, en cierto modo, provoca una "sobre protección" del espacio y consecuentemente una gestión no siempre bien coordinada entre las diversas administraciones en materia medioambiental, $\mathrm{y}$, sobre todo, no es bien entendida por la población afectada.

\section{Conclusiones}

Llegados a este punto, se podría decir que la puesta en marcha y la aplicación de la legislación en materia de protección medioambiental que ha afectado al territorio de Castilla-La Mancha ha evolucionado al hilo de las circunstancias históricas y de los modelos dominantes en materia de protección medioambiental. La historia de las declaraciones de ENP en esta Comunidad Autónoma ha seguido muy de cerca las directrices teórico-conceptuales de los modelos de valoración y de selección de los ecosistemas para ser considerados ENP. En este sentido, se han aplicado en el transcurso de sus declaraciones los mismos criterios para selección de espacios que encontramos a grandes rasgos en el resto del Estado español.

Así, el elenco de declaraciones previas al año 1978, había seguido muy de cerca el mismo programa que hasta entonces había aplicado atendiendo a cánones de elección de espacios (rareza, peculiaridad, etc.) y que se habían materializado en los Sitios Naturales de Interés Nacional (Jiménez García-Herrera, 2000). En el caso de la Ciudad Encantada, atendía a las formas caprichosas y sorprendente del roquedo (calizas) (García Marchante, 2011); y en el caso de las lagunas de Ruidera en el territorio limítrofe de las provincias de Ciudad Real y Albacete, los criterios pretendían proteger un ecosistema frágil desde el punto de vista hídrico (problemas de suministro y mantenimiento de las láminas de agua, etc.).

Pero este planteamiento, en vigor desde principios del siglo XX hasta la Ley 15/1975, de 2 de mayo, propia de Espacios Naturales Protegidos, se ha ido transformando como consecuencia del traspaso de competencias en materia de protección de la naturaleza a las Comunidades Autónomas y fruto de ello, ha sido el aumento considerable de nuevos ENP a raíz de la normativa propia que ha supuesto, en términos de superficie, un incremento considerable. La nueva normativa (Ley 9/1999, de 26 de mayo) entre sus objetivos principales ha primado conseguir una protección lo más integral y holística posible recogiendo ejemplos de todos los ecosistemas hallados en la Región. Esta protección global ha pretendido proteger aspectos tan diversos como los cursos altos de algunos ríos (Alto Tajo, río Cuervo, 
Calares del río Mundo, etc.) así como también otros elementos específicos del relieve regional (bonales ${ }^{14}$, maares ${ }^{15}$, formaciones geológicas, etc.) mediante la aplicación de las distintas figuras que recoge la Ley. De esta forma, en el momento actual, los criterios de selección y declaración han cambiado desde la norma nacional de 1916 (primera Ley de Parques Nacionales) gracias a la incorporación de esta norma subrayando entre las decisiones en la elecciones de nuevos espacios aspectos como el alto valor medioambiental de algunos espacios por su buen estado de conservación, la excepcionalidad paisajística fundamentalmente natural y la importancia ecológica por la singularidad de algunos ecosistemas.

Con ello, se ha conseguido un objetivo fundamental: la distribución territorial más equilibrada de los ecosistemas de Castilla-La Mancha aunque para ello se haya tenido que esperar hasta la asunción de competencias por parte de la Administración Regional. Básicamente hasta ese momento (año 1978) dominaban dos tipos de espacios protegidos: humedales (Tablas de Daimiel, Lagunas de Ruidera) y ecosistemas relictos y en extinción (Hayedo de Tejera Negra). Aunque a este doble modelo se podrían añadir otro tipo de protección, de tintes productivistas y cinegéticos, que abarcaba amplios territorios considerados ideales para la práctica de la caza como fueron las reservas nacionales de Caza (Sonsaz, Serranía de Cuenca, Tablas de Daimiel, etc.) y del parque cinegético experimental del Hosquillo. Pero conforme se ha ido consolidando el modelo autonómico (años 1999-2000) se añadieron nuevos criterios de protección para abarcar los ecosistemas de bosque mediterráneo (Cabañeros), las formaciones geológicas (Hoces del Cabriel), humedales y lagunas (Laguna del Hito, Complejos lagunares,...), y volcanes inactivos, entre otros, consiguiendo así extender los criterios de protección a los diversos elementos naturales, geológicos y del paisaje del territorio castellano-manchego.

Esto implica que la visión puntual de lo valioso, seguida en la selección de los primeros espacios naturales protegidos, ha sido progresivamente sustituida por una visión sistemática y globalizada del territorio, que permite detectar y, por tanto, elegir una muestra de los ecosistemas y geo-sistemas más representativos tras sopesar criterios no sólo naturales sino también territoriales, económicos, sociales, jurídicos y administrativos. En este contexto, la extraordinaria debilidad de la red española de espacios protegidos hasta la configuración del estado autonómico provocaría, ya desde los inicios del nuevo marco político, una reacción protectora igualmente excepcional, de la que es buena muestra la comunidad castellano-manchega y la puesta en marcha de todo el aparato legislativo autonómico.

El resultado de todo esta normativa de aplicación en los últimos treinta años ha tenido su reflejo en la gestación de la Red de ENP de Castilla-La Mancha con más de 110 espacios protegidos lo que supone unas 600.000 ha. Sin duda alguna, desde la

14 También llamados bohonales o trampales. Son lugares con humedad permanente o casi permanente en donde el agua empapa el terreno o circula muy lentamente por la superficie formando pozas (AA. VV., 2003: 4).

15 Se trata de un vocablo de origen alemán que define aquellos cráteres explosivos originados a partir de erupciones volcánicas muy violentas (AA. VV., 2000: 694). 
puesta en marcha de la Ley autonómica se ha apoyado y favorecido enormemente la creación de nuevos parques naturales que son, a fin de cuentas, las figuras de mayor rango protector en el territorio considerándose la figura estrella, de forma que más del 50 \% de la superficie de esta Red está cobijada bajo esta figura mientras el uso de otras figuras de protección (paisaje protegido y paraje protegido) prácticamente ha sido testimonial.

Así mismo, algunos de estos ENP se incluyen en varias categorías de protección, no sólo ya de ámbito regional sino nacional e internacional (humedales Ramsar, Reserva de la Biosfera, Red Natura 2000, etc.) con el consiguiente riesgo de "sobre protección" y de una posible falta de coordinación entre las administraciones competentes para la correcta gestión de dichos espacios.

En cualquier caso, y a pesar de la multitud de figuras y la extensión que suponen las mismas, la protección de la naturaleza no debería reducirse a la mera preservación de los ecosistemas hasta convertirlos en un producto de la musealización del espacio. La política ambiental, al margen de la cantidad de declaraciones procedentes de distintos niveles administrativos (europeo, nacional, autonómico, etc.) debe apostar por el mantenimiento de los recursos y de los procesos naturales, dejando de lado la mera delimitación de espacios como islas gestionadas sin tener en cuenta su contextualización e integración con el entorno más amplio al que pertenecen (Casado de Otaloa, 1990).

En el análisis que se ha planteado, la configuración de la Red de ENP estaría dominada por dos grandes modelos: aquéllos ENP de superficie reducida y caracterizados por ser ecosistemas de humedales continentales situados en territorios con densidades medias de población, con cierta estabilidad y presencia significativa de pequeñas ciudades con dinamismo demográfico y poblacional considerable; y los ENP situados en áreas rurales con fuertes condicionantes naturales y geográficos, con dificultades de vertebración del territorio (comunicaciones, etc.) y bajísimas densidades de población que las convierten en auténticos "vacíos" poblacionales situándose preferentemente en espacios de montaña y respondiendo a ecosistemas de montaña mediterránea y formaciones cársticas. En el primero de los casos, cabría incluir los ENP situados en la zona central y oeste de Castilla-La Mancha (Tablas de Daimiel, complejos lagunares, Lagunas de Ruidera, etc.), mientras que en el segundo modelo se incluirían, los ENP correspondientes a las áreas rurales con baja presencia humana y que, o bien, bordean la Comunidad Autónoma (sistemas montañosos como el sistema Central, el sistema Ibérico, las sierras Béticas y sierra Morena), o bien se encuentran en el interior de la Castilla-La Mancha (los montes de Toledo o la sierra de Altomira). En estos territorios encontramos los parques naturales más extensos de la Red (Alto Tajo, Serranía de Cuenca, Valle de Alcudia o Cabañeros, entre otros) y además de un buen conjunto de otras figuras de protección (monumentos, reservas, microrreservas, reservas fluviales, etc.). La escasa presencia humana en estas comarcas ha ayudado al mantenimiento de los altos niveles de calidad de los ecosistemas de muchos de estos espacios naturales. Por el contrario, son áreas que acarrean problemas estructurales (falta de población joven, envejecimiento acusado, 
pérdida de servicios mínimos, etc.) que proyectan en el ENP una esperanza como reclamo turístico para la comarca apostando por el turismo rural y de naturaleza para completar sus rentas agroganaderas (Vacas Guerrero, 2003, Muñoz Flores, 2006), hecho que especialmente de deja notar en la comarca de Cabañeros (AA.VV., 2005). Este aspecto lleva a pensar en la necesidad de establecer una relación estrecha entre los ENP y el desarrollo rural y los efectos socioeconómicos en los territorios (Múgica De La Guerra, Fernández Sañudo et al., 1998) percibiendo en estas nuevas figuras de protección una posibilidad más para salir del estancamiento demográfico y social en que se encuentran (Tolón Becerra, 2000) aunque no siempre se consiga con éxito este cometido surgiendo conflictos territoriales (Troitiño Vinuesa, 1995) y teniendo en cuenta que para conseguir este objetivo es fundamental superar el posible rechazo social que pueda surgir a raíz de la declaración de un nuevo espacio protegido (Quiroga, 1997).

Así mismo, encontramos pues una fuerte polarización entre los ENP declarados a raíz de la normativa regional. Por un lado, aquellos que, fundamentalmente bajo la figura de parque natural, se caracterizan por su extensión: la Serranía de Cuenca, el Alto Tajo, el Barranco del río Dulce, la Sierra Norte de Guadalajara, el Valle de Alcudia, etc., con la salvedad del monumento natural de Tierra Muerta y Palancares; y por otro, ENP de reducidas dimensiones: hoz de Beteta, laguna del Prado, cueva de los Murciélagos, etc., que generalmente serán amparados por figuras como reserva natural o microrreserva y en los que dominan los criterios geomorfológicos, hidrológicos y biogeográficos. Este tipo de ENP ha proliferado mucho en los últimos años frente a la utilización de otra figura que suele proteger espacios de extensión media como es la de monumento natural.

No obstante, y a pesar de ese espíritu de protección holística que persigue la normativa, no todos los territorios gozan del mismo nivel de consideración y protección. La Mancha, escenografía de un paisaje literario (García Marchante y Fernández Fernández, 2000), compartida por cuatro provincias, carece de protección paisajística en un sentido integral como también le ocurre a la Alcarria. A lo sumo, ambos territorios se han visto afectados por figuras de diversa categoría, que ha ido protegiendo y tratando de conservar elementos naturales como la riqueza hídrica y geomorfológica que encontramos en su territorio: lagunas -de diferentes orígenes-, tablas de aguas, navas, saladares, maares, surgencias, etc.; todo ello fruto de la orogenia que afectó a los materiales que forman el zócalo precámbrico de la meseta y el particular comportamiento de los materiales sedimentarios frente a la erosión que originaría tal diversidad de formas del relieve. No obstante, salvo la figura de parque nacional (Tablas de Daimiel) o regional (parque natural Lagunas de Ruidera), o las distintas microrreservas existentes, otras figuras de escala internacional (Reserva de la Biosfera) parecen haberse convertido en una etiqueta de calidad y de prestigio para los espacios seleccionados antes que desarrollarse como una normativa estricta a cumplir, dejando de lado, en algunas ocasiones, los objetivos y finalidades para las que fueron creados. 


\section{Bibliografía}

AA.VV. (2003). Bonales, bohonales o trampales. Humedales poco conocidos. Revista Medio Ambiente Castilla-La Mancha, 11, 4-13.

AA.VV. (2005). Atlas de Turismo Rural de Castilla-La Mancha. Madrid, CNIG, Ministerio de Educación y Cortes de Castilla-La Mancha.

Casado de Otaloa, S. (1990). Pioneros de la conservación de la naturaleza en España. Quercus, 70, 32-38.

Donaire Jiménez, C. (2005). La protección de los ecosistemas y los espacios naturales protegidos en Castilla-La Mancha. Boletín de la Sección del Estado Español de EUROPARC, 19, 33-38.

García Marchante, J. S. (2011). La Ciudad Encantada: de museo vivo de geología a icono turístico conquense. Actas del XII Coloquio de Geografía del Turismo, Ocio y Recreación, 237-245.

García Marchante, J. S. y FERNÁNDEZ FERNÁNDEZ, M.C. (2000). El espacio del Quijote. El paisaje de la La Mancha. Cuenca, Universidad de Castilla-La Mancha.

García Rayego, J. L. (2001). Paisaje de espacios naturales protegidos de Castilla-La Mancha. En Pillet Capdepón, F. y Plaza Tabasco, J. (Ed.): Lecciones de Desarrollo Rural. Una aproximación formativa desde y para Castilla-La Mancha. Ciudad Real, Universidad de Castilla-La Mancha, 247-250.

García Rayego, J. L. (1995). Políticas recientes de conservación del medio natural en CastillaLa Mancha. XIV Congreso Nacional de Geografía. A.G.E. y Departamento de Geografía de la Universidad de Salamanca, 29-34.

García Rayego, J. L. y SERRANO DE LA CRUZ SANTOS-OLMO, M. A. (2007). Los Espacios Naturales Protegidos de Castilla-La Mancha. En Pillet Capdepón, F. (ed.) Geografía de Castilla-La Mancha. Ciudad Real, ALMUD, 119-138.

Gómez Mendoza, J. (1999). Paisaje y Espacios Naturales Protegidos en España. El Boletín de la Institución Libre de Enseñanza, 34-35, 131-152.

Gómez Orea, D. y Rubio Blanco, D. (2000). Plan de ordenación de los recursos naturales y desarrollo sostenible del Alto Tajo. Observatorio Medioambiental, 3, 159-194.

Jiménez García-Herrera, J. (2000). La situación jurídica de los espacios naturales protegidos. En González Martín, J. A. y Vázquez González, A. (ed.) Guía de los Espacios Naturales de Castilla-La Mancha. Toledo, Servicio de Publicaciones de la Junta de Comunidades de Castilla-La Mancha, 101-117.

Martín Herrero, J. (1995). La protección de los espacios naturales y la vida silvestre en Castilla-La Mancha. Montes, 39, 22-30.

Martín Herrero, J. (2003). La vegetación protegida en Castilla-La Mancha: descripción, ecología y conservación de los hábitat de protección especial. Toledo, Servicio de Publicaciones de la Junta de Comunidades de Castilla-La Mancha.

Mata Olmo, R. (2011). Tipos y asociaciones de tipos de paisaje de Castilla-La Mancha: un repertorio paisajístico de interior ibérico. En AA.VV. (ed.) Atlas de los Paisajes de Castilla-La Mancha. Cuenca, Universidad de Castilla-La Mancha. 
Múgica de la Guerra, M., Fernández Sañudo, P. y De Lucio Fernández, j.V. (1998). Efectos socioeconómicos de los Espacios Naturales Protegidos Contribución al debate. Actas del $4^{\circ}$ Congreso EUROPARC-ESPAÑA. EUROPARC-España, 23-32.

Mulero Mendigorri, A. (2002). La protección de espacios naturales en España: antecedentes, contrastes territoriales, conflictos y perspectivas. Madrid, Mundi-Prensa.

Muñoz Flores, J. C. (2006). Turismo y sostenibilidad en espacios naturales protegidos. Sevilla, Fundación Biodiversidad y Ministerio de Medio Ambiente.

Quiroga, A. (1997). Aceptación de los espacios protegidos por la población local. Seminario sobre Alternativas de Desarrollo Económico para la Población Local de los Espacios Naturales Protegidos. Madrid, Fundación Fernando Goonzález Bernáldez, 18-20.

Ruiz Martínez, S. (2001). Los espacios naturales protegidos y la ley 9/1999, de 26 de mayo, de conservaión de la naturaleza, de Castilla-La Mancha. Revista jurídica de Castilla-La Mancha, 30, 295-316.

Ruiz Pulpón, Á. R., Serrano de la Cruz, M. A y Jeréz, Ó. (2010). Repercusiones de las políticas públicas en la transformación de espacios de alto valor ambiental: contradicciones en La Mancha Húmeda. En: Cebrián, F; Pillet, F. y Carpio, J. (ed.) Las escalas de la Geografía: del mundo al lugar. Homenaje al profesor Miguel Panadero Moya. Cuenca, Servicio de Publicaciones de la Universidad de Castilla-La Mancha, 303-328.

Sánchez Soler, M. J. y Del Moral Fernández del Rincón, A. (2000). El parque nacional del las Tablas de Daimiel. En González Martín, J. A. y Vázquez González, A. (ed.) Guía de los Espacios Naturales de Castilla-La Mancha. Toledo, Servicio de Publicaciones de la Junta de Comunidades de Castilla-La Mancha, 481-494.

Serrano de la Cruz Santos-Olmo, M. A. (2014). La Red de Áreas Protegidas de Castilla-La Mancha: los Espacios Naturales Protegidos y las Zonas Sensibles. En Jeréz García, Ó y Rodriguez Domenech, M.A. Las Áreas Protegidas de Castilla-La Mancha. Tic y bilingüismo como recursos didácticos para la Formación Profesional. Ciudad Real, Óptima diseño e impresión, 83-134.

Serrano Gil, Ó. (2009). Espacios naturales protegidos en territorios de ruralidad profunda: el caso del parque natural del Alto Tajo. Actas del XXI congreso de geógrafos españoles. Geografía, territorio y paisaje: el estado de la cuestión, 1641-1658.

Serrano Gil, Ó. y Vázquez Varela, C. (2007). Historia de los Espacios Naturales Protegidos en Castilla-La Mancha (1927-2007). III Coloquio Hispano-Francés de Geografía Rural, 138163.

Tolón Becerra, A. (2000). Espacios naturales protegidos. Desarrollo rural y socioeconómico. Agricultura: Revista agropecuaria, 813, 166-170.

Troitiño Vinuesa, M. Á.(1995). Espacios naturales protegidos y desarrollo rural: una relación territorial conflictiva. Boletín de la Asociación de Geógrafos Españoles, 20, 7-37.

Vacas Guerrero, T. (2003). Recursos territoriales turísticos: los espacios naturales protegidos españoles. Madrid, Universidad Rey Juan Carlos. 


\section{Fuentes}

Jefatura del Estado (1975). Ley 15/1975, de 2 de mayo, de Espacios Naturales Protegidos.

Jefatura del Estado (1982). Real Decreto 1105/1982, de 14 mayo, de actuación del ICONA en las zonas de influencia socio-económica.

Jefatura del Estado (1984). Real Decreto 1676/1984, de 8 de febrero, traspaso de funciones y servicios del Estado en materia de conservación de la naturaleza.

Jefatura del Estado (1989). Ley 4/1989, de 27 de marzo, de Conservación de los Espacios Naturales y de la Flora y Fauna Silvestre.

Presidencia de Junta de Comunidades de Castilla-La Mancha (1999). Ley 9/1999, de 26 de mayo, de Conservación de la Naturaleza.

Ministerio de Agricultura, Alimentación y Medio Ambiente (2014): Resolución de 17 de noviembre de 2014, de Parques Nacionales, por la que se publica la aprobación por la UNESCO de la ampliación de la Reserva de la Biosfera de Montseny, Cataluña, y la Reserva de la Biosfera de La Mancha Húmeda, en Castilla-La Mancha.

\section{Recursos web}

www.ine.es

www.ies.jccm.es

http://www.magrama.gob.es/es/

http://www.cajaespana-duero.es/obrasocial 